\title{
THE UTILITY OF POSITRON EMISSION TOMOGRAPHY FOR THE DIAGNOSIS AND STAGING OF RECURRENT ESOPHAGEAL CANCER
}

P. Flamen, MD

A. Lerut, $\mathrm{MD}, \mathrm{PhD}$

E. Van Cutsem, MD, $\mathrm{PhD}$

J. P. Cambier, MD

A. Maes, MD, $\mathrm{PhD}$

W. De Wever, MD

M. Peeters, MD, PhD

P. De Leyn, MD, PhD

D. Van Raemdonck, MD, PhD

L. Mortelmans, MD, PhD
Objective: To study the utility of whole-body positron emission tomography with ${ }^{18}$ F-fluoro-deoxy-D-glucose (FDG-PET) for the evaluation of recurrence after curative resection of cancer of the esophagus or gastroesophageal junction.

Methods: Forty-one patients with a clinical or radiologic suspicion of recurrent disease underwent conventional diagnostic work-up, including a spiral computed tomographic scan, an endoscopic ultrasound, and a dedicated whole-body FDG-PET. PET lesions were classified as equivocal or suspicious recurrence. The conventional diagnostic work-up and PET findings were correlated with pathology or with radiologic and clinical follow-up. Equivocal lesions were classified as positive.

Results: Forty recurrences were found in 33 patients. The lesions were perianastomotic $(n=9)$, regional $(n=12)$, and at distant sites $(n=19)$. For the diagnosis of a perianastomotic recurrence, the sensitivity, specificity, and accuracy of FDG-PET were $100 \%, 57 \%$, and $74 \%$, versus $100 \%, 93 \%$, and 96\% for conventional diagnostic work-up, respectively $(P=$ not significant). False-positive PET lesions were found in patients with a progressive anastomotic stenosis requiring repetitive endoscopic dilatation. For the diagnosis of regional and distant recurrences, the sensitivity, specificity, and accuracy of PET were $94 \%, 82 \%$, and $87 \%$, versus $81 \%(P=$ not significant $), 82 \%(P=$ not significant $)$, and $81 \%(P=.0771)$ for conventional diagnostic work-up. All false-positive PET lesions $(n=4)$ had been reported as equivocal. On a patient base, PET provided additional information in 11 of $41(27 \%)$ patients. A major impact on diagnosis was found in 5 patients with equivocal or negative findings on complete diagnostic workup in whom PET provided a true-positive diagnosis. In 5 other patients the diagnosis was staged upward from localized to extended recurrent disease, and in 1 patient with an equivocal complete diagnostic work-up, PET correctly excluded malignancy.

Conclusion: FDG-PET allows a highly sensitive diagnosis and accurate whole-body staging of symptomatic recurrent esophageal cancer. Further studies in asymptomatic patients are needed to assess the potential benefit on survival. (J Thorac Cardiovasc Surg 2000;120:1085-92)
From the Departments of Nuclear Medicine, Internal Medicine, Radiology, and Thoracic Surgery, University Hospital Gasthuisberg, Katholieke Universiteit Leuven (KUL), Leuven, Belgium.

Received for publication April 4, 2000; revisions requested May 22, 2000; revisions received June 12, 2000; accepted for publication July 27, 2000.

Address for reprints: Patrick Flamen, MD, Department of Nuclear Medicine, University Hospital Gasthuisberg, Herestraat 49, 3000 Leuven, Belgium (E-mail: Patrick.Flamen@uz.kuleuven.ac.be).

Copyright (C) 2000 by The American Association for Thoracic Surgery

0022-5223/2000 $\$ 12.00+0 \quad \mathbf{1 2 / 1 / 1 1 0 4 6 4}$

doi:10.1067/mtc.2000.110464 ong-term survival after cancer of the esophagus or L the gastroesophageal junction remains poor despite significant advances in surgical techniques and perioperative management. ${ }^{1-4}$ Even after apparently curative surgery, the overall 5-year survival is only $30 \%$ to $50 \%$. In approximately one third of the patients, the recurrence is located in the operative field. The lesions are usually seen in regional lymph nodes or as a mass originating in the mediastinum and infiltrating the gastric pull-up from outside. The majority of the recurrences, however, are distant metastases, indicating the systemic 
character of the disease. ${ }^{5-7}$ Two thirds of patients have a recurrence within 1 year and nearly all within 2 years after the primary operation. ${ }^{8}$ Nevertheless, most centers do not actively search for tumor recurrence in asymptomatic patients because the survival benefit of such an approach remains controversial. Some reports, however, suggested that early detection of recurrent disease is desirable because aggressive treatment may result in prolonged tumor-free survival or occasional cure. ${ }^{9}$ The conventional diagnostic work-up (CDW) currently available for the postoperative diagnosis and staging of recurrent disease includes endoscopy, transesophageal endoscopic ultrasound (EUS), and computed tomography (CT) of the chest and abdomen. A fundamental limitation of these techniques is that they depend entirely on anatomic and/or structural criteria for diagnosis. Therefore, their specificity is hampered by therapy-induced changes of the tissue characteristics at the operative site. ${ }^{10}$ Moreover, strictures at the esophagogastric anastomosis often preclude full passage of the endoscope, resulting in incomplete diagnosis in one third of the patients. ${ }^{11}$

Recent reports indicated a role for whole-body positron emission tomography (PET) using the radiolabeled glucose analog ${ }^{18}$ F-fluoro-deoxy-D-glucose (FDG) for preoperative staging of cancer of the esophagus and gastroesophageal junction. ${ }^{12-16}$ PET uses a metabolic approach to diagnosis. The depiction of neoplastic foci by FDG-PET relies on the increased accumulation of the radioactive tracer in malignant tissues, which is believed to be the result of an increased expression of glucose transport enzymes in the tumoral cell membrane, together with an increased activity of the enzymes of the first steps of the glycolytic pathway. ${ }^{17,18}$ Early reports suggested that PET had a significant advantage over the conventional methods (spiral CT and EUS) for the detection of distant metastases and thus for the diagnosis of nonresectable disease. For assessing locoregional lymph node metastases, PET was as accurate as CDW and more specific. No data are yet available about the potential of PET used in the follow-up of patients after resection of esophageal cancer. The purpose of this study was to investigate the utility of FDG-PET for the diagnosis and staging of disease in patients in whom recurrent esophageal cancer was suspected.

\section{Methods}

Patients. The database of the PET department was searched for all in-house patients who underwent a wholebody FDG-PET scan for clinical and/or radiologic suspicion of recurrent esophageal cancer during the period January
1998 until June 1999. Only patients who had undergone an esophagectomy with curative intent were selected.

CDW. In all patients routine CDW was performed in house according to high-standard practice, including spiral CT of chest and abdomen $(n=39)$, transesophageal EUS $(n=13)$, and endoscopy $(n=18)$. In some patients, other sign- or symptom-directed dedicated techniques such as bone scan or spiral CT of the neck were also performed. In 11 patients full passage of the echoendoscope was impossible, precluding accurate EUS diagnosis. These patients underwent an endoscopy. The lesions reported by CDW were classified as equivocal or suspicious for recurrence.

FDG-PET imaging. The PET imaging was performed with a CTI-Siemens 931 or HR+ scanner (CTI, Inc, Knoxville, Tenn) with an axial field of view of $10.1 \mathrm{~cm}$ and $15 \mathrm{~cm}$, respectively, and a spatial resolution of 8 and $6 \mathrm{~mm}$, respectively. All patients fasted for 6 hours before the tracer administration. Sixty minutes after the intravenous injection of a $6.5 \mathrm{MBq} / \mathrm{kg}$ dose of ${ }^{18} \mathrm{~F}-\mathrm{FDG}$ (to a maximum of 555 $\mathrm{MBq}$ ), a whole-body emission scan was performed. The raw imaging data were reconstructed in a $128 \times 128$ matrix with the use of an iterative reconstruction algorithm.

For the purpose of this study, all PET images were reviewed by two experienced nuclear medicine physicians (A.M. and J.P.C.). At the time of image review, these observers were fully aware of the history of the patients, the type of esophagectomy performed, the location of the esophagogastric anastomosis, and the patients' symptoms. However, the observers were completely blinded to the results of the concurrent $\mathrm{CDW}$ and to the follow-up data. In case of diagnostic discordances between the observers, a consensus diagnosis was generated. The PET lesions were classified as equivocal or suspicious for recurrence.

Data analysis. The results of CDW and of FDG-PET were compared with a gold standard that consisted of histologic findings or of clinical and radiologic follow-up. A follow-up of at least 6 months was needed for a definitive negative diagnosis.

For the region-based analysis, recurrences were assigned to 3 regions: (1) the perianastomotic area, located around the esophagogastric anastomosis; (2) the regional area, corresponding to the operative field of the primary esophagectomy, including the mediastinum and upper abdominal compartment, and also the cervical area when a 3-field lymphadenectomy had been performed; and (3) distant areas, if the lesions were located in organs or lymph nodes outside the initial operative field.

The calculations of the accuracies of CDW and PET took into account only those regions for which a gold standard was available. For these calculations, CDW and PET lesions that were classified as equivocal were considered as positive.

The sensitivity, specificity, and accuracy of CDW and FDG-PET were calculated by the standard definitions. McNemar $\chi^{2}$ testing was used to compare the sensitivity, specificity, and accuracy of PET and CDW as dependent samples by means of $2 \times 2$ tables. 
Table I. Patients' characteristics

\begin{tabular}{lc}
\hline Characteristics & No. \\
\hline Demographics & \\
Mean age (standard deviation) & $62(9)$ \\
Male/female & $36 / 5$ \\
Primary esophageal tumor & \\
Adenocarcinoma/spinocellular carcinoma & $27 / 14$ \\
Location & \\
$\quad$ Proximal & 3 \\
Middle & 8 \\
$\quad$ Distal & 12 \\
GE junction & 18 \\
Primary curative surgery & \\
Partial esophagogastrectomy & \\
With cervical anastomosis & 35 \\
$\quad$ With intrathoracic anastomosis & 5 \\
Endoscopic mucosectomy & 1 \\
pTNM & \\
Stage 1 & 6 \\
Stage 2 & $57 / 3 / 2$ \\
Stage 3 & \\
Stage 4 & 5 \\
Invaded surgical resection margins & 14 \\
Ro/R / ${ }_{2}$ resection & 16 \\
\hline
\end{tabular}

\section{Results}

Patients. Forty-one patients were included for analysis. Table I lists the patients' demographics, primary esophageal tumor type and location, type of operation that was performed on the primary tumor, pathologic TNM staging (pTNM), and the location of the esophagogastric anastomosis.

The suspicion of tumor recurrence was based on the presence of dysphagia $(n=8)$, hoarseness $(n=8)$, pain $(n=9)$, deterioration of the general condition $(n=11)$, or abnormalities detected during routine clinical or radiologic examinations $(n=5)$. The mean time elapsed between the date of the primary operation and the date of the PET scan was 506 days (range 54-2151 days).

The gold standard established cancer recurrence in 33 of $41(80 \%)$ patients. Table II shows the regional distribution of the recurrences and the gold standard used to define the true lesion status. The sensitivity, specificity, and accuracy of CDW and FDG-PET for the diagnosis of perianastomotic recurrence, locoregional recurrence, and distant metastasis are shown in Table III. Overall, sensitivity of FDG-PET was higher than that of CDW (95\% vs $85 \% ; P=$ not significant [NS]). The specificity, on the other hand, was lower (72\% vs $86 \% ; P=\mathrm{NS}$ ). This resulted in a similar overall accuracy of the two diagnostic approaches (84\% vs $86 \%$ for PET and CDW, respectively).
Table II. Distribution of the recurrences and the methods used to define the true lesion status (gold standard)

\begin{tabular}{lccc}
\hline & & \multicolumn{2}{c}{ True lesion status defined by: } \\
\cline { 3 - 4 } & $\begin{array}{c}\text { No. of } \\
\text { recurrences }\end{array}$ & Histology & $\begin{array}{c}\text { Follow-up } \\
\text { (mean duration)* }\end{array}$ \\
\hline Anastomosis & 9 & 8 & 1 (102 days) \\
Regional & 12 & 7 & 5 (122 days) \\
Distant & 19 & 8 & $11(105$ days) \\
Total & 40 & $23(58 \%)$ & $17(42 \%)$ \\
\hline
\end{tabular}

*The mean duration represents the mean time elapsed between the date of the PET and the clinical and/or radiographic confirmation of the true-lesion status.

Perianastomotic recurrence. The gold standard established perianastomotic recurrent disease in 9 of 41 (22\%) patients. Both CDW and PET detected all the lesions, resulting in a $100 \%$ sensitivity for both approaches. A definite negative diagnosis was made in 14 patients. This patient subset was used for calculation of the specificity. The specificity of PET was lower than that of $\mathrm{CDW}$ (57\% vs $93 \%$, respectively) due to false-positive PET lesions found in 6 of 14 patients. These lesions were classified as suspicious in 3 patients and as equivocal in 3 other patients. A review of the records of these patients revealed that the former 3 patients had undergone an endoscopic dilation treatment for a benign stricture 7, 14, and 55 days, respectively, before the PET scan was performed. In 2 of these patients the dilatation was performed less than a week before the PET scan and in the other patient, 55 days before the PET scan. An illustrative example of a PET lesion falsely classified as suspicious for recurrence at the perianastomotic site is shown in Fig 1. The 3 other patients had only moderately intense lesions, and because of this their status was classified as equivocal. In 2 of these patients endoscopy revealed a stricture of the anastomosis, without a recent therapeutic dilation. In the third patient the time interval between the primary operation and the PET scan was only 54 days. The lesion probably reflected postoperative inflammatory healing processes.

Regional recurrence. The gold standard indicated a regional recurrence in 12 patients and the absence of recurrent disease in 12 patients. CDW had a lower sensitivity than PET (83\% and $92 \%$, respectively) and a higher specificity (92\% and $83 \%$, respectively), resulting in a minimally different accuracy $(83 \%$ and $88 \%$, respectively). One false-negative PET was found in a patient with a biopsy proven recurrent tumor of more than $2 \mathrm{~cm}$ in diameter located in the upper retroperi- 


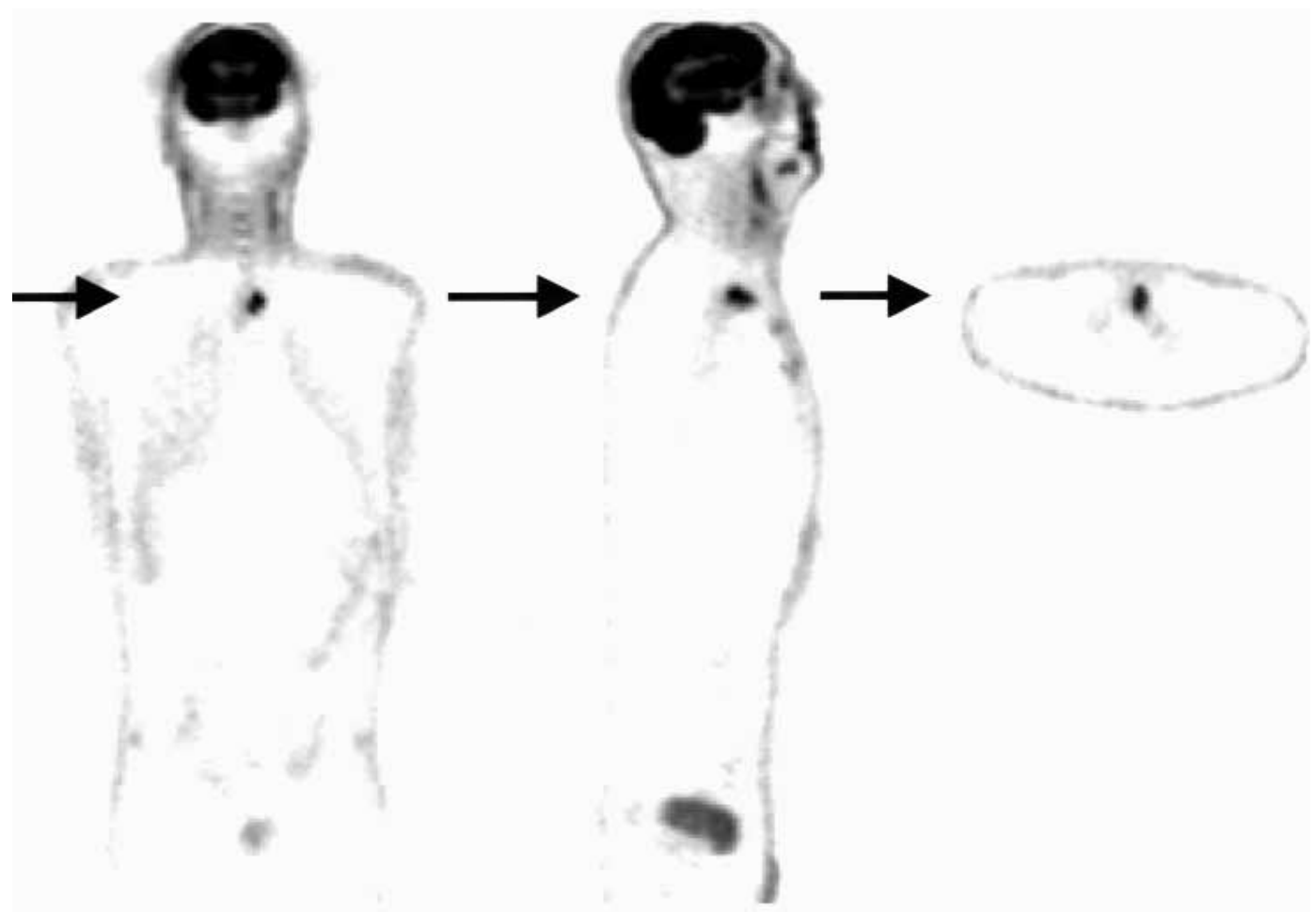

Fig 1. Example of a false-positive FDG-PET finding at the anastomosis. This patient had dysphagia 1 year after a subtotal esophagogastrectomy and cervical esophagogastrostomy. Endoscopy indicated a benign intrinsic stenosis of the esophagogastric anastomosis and an endoscopic dilatation was succesfully performed. Two weeks later the PET scan showed an intense focal hot spot in the upper mediastinum. Seven months' clinical and radiologic follow-up did not show any signs of recurrent disease.

Table III. Regional based analysis

\begin{tabular}{|c|c|c|c|c|c|c|c|c|}
\hline \multirow[b]{2}{*}{ Region of recurrence } & \multicolumn{2}{|c|}{ Gold standard } & \multicolumn{3}{|c|}{$C D W$} & \multicolumn{3}{|c|}{$F D G-P E T$} \\
\hline & + & - & Sensitivity & Specificity & Accuracy & Sensitivity & Specificity & Accuracy \\
\hline \multirow[t]{2}{*}{ Anastomosis } & 9 & 14 & $9 / 9$ & $13 / 14$ & $22 / 23$ & 9/9 & $8 / 14$ & $17 / 23$ \\
\hline & & & $100 \%$ & $93 \%$ & $96 \%$ & $100 \%$ & $57 \%$ & $74 \%$ \\
\hline \multicolumn{9}{|l|}{ Regional } \\
\hline & 12 & 12 & $10 / 12$ & $11 / 12$ & $21 / 24$ & $11 / 12$ & $10 / 12$ & $21 / 24$ \\
\hline & & & $83 \%$ & $92 \%$ & $88 \%$ & $92 \%$ & $83 \%$ & $88 \%$ \\
\hline \multicolumn{9}{|l|}{ Distant } \\
\hline & 19 & 10 & $15 / 19$ & $7 / 10$ & $22 / 29$ & $18 / 19$ & $8 / 10$ & $26 / 29$ \\
\hline & & & $79 \%$ & $70 \%$ & $76 \%$ & $95 \%$ & $80 \%$ & $90 \%$ \\
\hline \multirow[t]{2}{*}{ All } & 40 & 36 & $34 / 40$ & $31 / 36$ & $65 / 76$ & $38 / 40$ & $26 / 36$ & $64 / 76$ \\
\hline & & & $85 \%$ & $86 \%$ & $86 \%$ & $95 \%$ & $72 \%$ & $84 \%$ \\
\hline
\end{tabular}

toneal area. A close review of the PET scan with visual registration of PET and CT images revealed only a faint tracer uptake at the site of the suspicious mass. The resected primary tumor was a pT3 N2 M0 adenocarcinoma of the gastroesophageal junction with a marked signet cell differentiation.
False-positive PET scans were found in 2 patients: both lesions were only moderately intense and were considered as equivocal. The first lesion was located in the anterior mediastinum and was not confirmed by follow-up CT performed 9 months later. The latter lesion was located in the right lung hilus. Histologic 


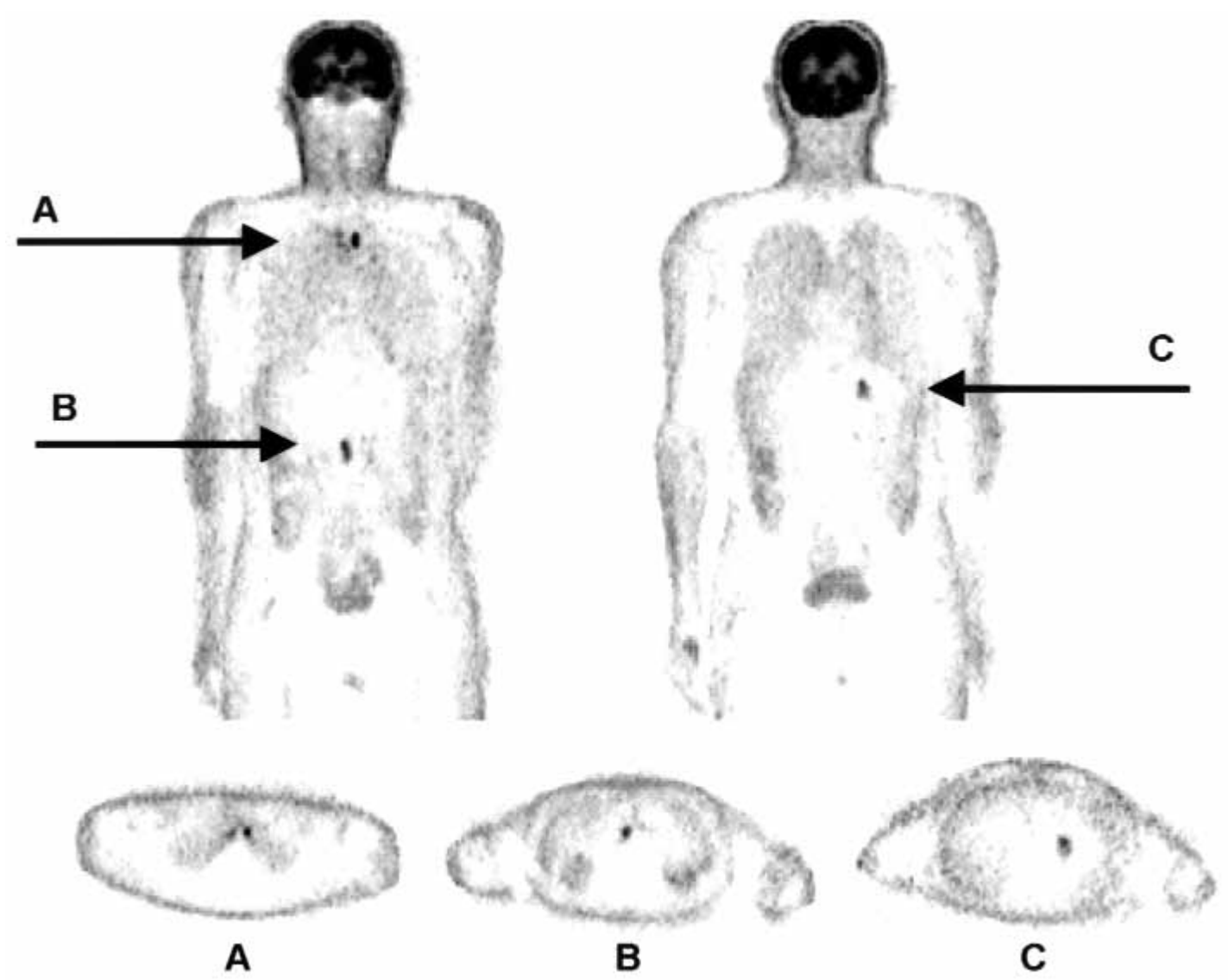

Fig 2. Example of a whole-body FDG-PET in a patient having hoarseness 6 months after a subtotal esophagogastrectomy and cervical esophagogastrostomy for a spinocellular epithelioma staged pT3 N1 Mb. CDW, including endoscopy and CT, did not demonstrate recurrent tumor. FDG-PET showed multiple sites of tumor recurrences in the upper mediastinum $(A)$, upper abdominal retroperitoneum $(B)$, and in the perigastric area $(C)$. All these lesions were confirmed by radiologic follow-up 3 months later.

examination of the hilar lymph nodes indicated inflammation.

Distant metastases. The gold standard indicated distant metastatic disease in 19 patients: CDW detected lesions in 15 (sensitivity 79\%) and FDG-PET in 18 (sensitivity 95\%) of these. The PET scan was falsenegative in only 1 patient, in whom diffuse miliary peritoneal metastases were found during a diagnostic laparoscopy. Conventional imaging was also false-negative in this patient.

False-positive PET images were found in 2 patients. One had a moderately intense FDG uptake in a spinal location, which was considered as equivocal. Other investigations and a disease-free follow-up of more than 1 year did not confirm the diagnosis of a bone metastasis. Another patient had a moderately intense lesion, also considered as equivocal, located in the periphery of the left lung. The CT of that region showed a thickened pleura and some parenchymatous sequelae of infection.

Regional and distant recurrences. For the diagnosis of a nonperianastomotic recurrence, that is, regional and distant, the sensitivity, specificity, and accuracy of PET were $94 \%, 82 \%$, and $87 \%$ versus $81 \%(P=$ NS), $82 \%(P=\mathrm{NS})$, and $81 \%(P=.0771)$ for CDW. Five lesions were classified as equivocal by PET: 2 regional and 3 distant lesions. The gold standard, available in 4 of them, indicated the absence of recurrent tumor. The 2 regional lesions were located in the lung hilus and in the anterior mediastinum. The 2 distant lesions were located in the lungs and in the bone. After close multidisciplinary correlation with clinical and/or radiographic findings, these lesions were considered as probably inflammatory and, therefore, did not induce 
any diagnostic or therapeutic change. Importantly, none of the equivocal PET lesions proved to be malignant.

Patient-based analysis. On a patient basis, PET and CDW were completely concordant in 25 of 41 $(61 \%)$ patients. Of the 16 patients with discordant PET findings, PET improved the diagnosis in 11 patients. According to the degree of potential clinical impact in these patients, the added value of PET was classified as major when a potential treatment could have been installed, withheld, or changed as a consequence of the PET findings; the value was classified as minor in case PET did not significantly change the management of the patient. A major contribution of PET was found in 5 of $41(12 \%)$ patients in whom it correctly diagnosed recurrent disease not detected by CDW. Fig 2 shows the FDG-PET images of such a patient. In 6 other patients, contribution of PET to the correct diagnosis was only minor: in 5 of these patients PET detected more lesions than CDW, and in another patient with equivocal CDW findings FDGPET correctly excluded a bone metastasis as the cause of a dorsal vertebral collapse.

On the other hand, PET had a potentially negative impact on diagnosis in 5 patients. PET falsely understaged the disease in 1 patient because of a false-negative finding, and it falsely overstaged the disease in 4 patients due to false-positive findings.

\section{Discussion}

Recently published studies investigated the usefulness of FDG-PET for primary staging of esophageal cancer. The diagnostic superiority of metabolism-based methods such as FDG-PET should be most pronounced in those circumstances in which nonspecific sequelae of surgery, radiotherapy, or chemotherapy render the conventional, anatomy-based modalities less accurate. This is substantiated by several reports on the successful use of FDG-PET for the metabolic imaging of perirectal masses often occurring after surgery of rectal adenocarcinomas and in the surveillance of patients operated on for head and neck carcinoma. ${ }^{19,20}$ The current study is the first that specifically focused on the utility of FDG-PET imaging for the diagnosis and staging of recurrent esophageal cancer. Our preliminary results suggest that FDG-PET provides a highly sensitive diagnosis (overall sensitivity 95\%) of recurrent disease, both locoregionally and at distant sites. However, in contrast to the reported findings in colorectal and head and neck cancer, ${ }^{19,20}$ PET does not seem to enhance the diagnostic accuracy for locoregional recurrences.

We believe that the inclusion criteria of this study led to an underestimation of the additional value of PET.
Only patients with a clinical or radiologic suggestion of recurrence were referred for a PET scan. The pre-test probability of finding recurrent tumor in these patients is very high. Indeed, $80 \%$ of the included patients had recurrent disease, often in advanced stages, leaving only the small fraction of CDW-negative patients to test the additional value of PET. Nevertheless, the very high sensitivity of PET supports the hypothesis that PET could allow an earlier and presumably preclinical diagnosis of esophageal cancer recurrence. It certainly justifies further prospective studies in this respect. Such studies have been reported using CT or EUS as surveillance tools in the year after the primary operation. In a prospective follow-up of patients with routine CT, Barbier and associates ${ }^{11}$ showed that the symptom-free interval exeeded the tumor-free interval by 4 months. However, the earlier detection of asymptomatic recurrences had no impact on outcome. More recently, EUS was tested in a similar setting. It was found that EUS performed routinely in the postoperative follow-up detected recurrences in $67 \%$ of the asymptomatic patients, on average 3 months before their condition became symptomatic. ${ }^{21}$ An important limitation of EUS is that a benign anastomotic stricture precluding passage of an endoscope after cervical esophagogastrostomy will develop in approximately $30 \%$ of patients. ${ }^{22}$

Whether earlier diagnosis of recurrent esophageal cancer would improve patient survival has not been reported yet. The possibility of a survival benefit, however, can be deduced indirectly from results obtained in a study with primary chemotherapy in asymptomatic patients with advanced colorectal cancer, indicating a survival benefit of 6 months. ${ }^{23}$ The available therapeutic modalities in recurrent esophageal cancer are radical reresection, palliative resection and bypass, laser thermocoagulation, stenting, chemotherapy, brachytherapy, and radiotherapy, alone or in combination. The choice of a specific therapeutic modality depends on the extent of the recurrence. Our study results indicate that FDG-PET at that point could contribute to patient care because it provides, in one imaging session covering the whole body, an accurate assessment of the extent of disease.

A noteworthy finding in the current study is the high incidence of false-positive PET findings at the perianastomotic region. By reviewing the patients' records, we found that the patients with intense false-positive FDG uptake all had undergone an endoscopic dilation of a benign anastomotic stricture as long as 55 days before the PET scan. Probably the dilation induced a trauma resulting in an inflammatory reaction. Falsepositive FDG uptake at inflammatory lesions is widely 
known and remains a major problem in the diagnosis of oncologic patients. ${ }^{24}$ Three other patients had only moderate uptake at the anastomosis. In 2 of these patients, a nontumoral stricture was found endoscopically. However, a dilatation had not been performed, and the accumulation of FDG may have been due to proliferating fibroblastic and/or granulomatous tissue at the anastomosis. The other patient underwent scanning less than 2 months after the primary operation. The FDG uptake in this patient probably reflected a normal inflammatory healing process. This means that interpretation of the PET scan at the anastomotic site needs careful correlation with clinical data before a positive diagnosis can be made. The specificity of PET at the regional and distant sites is clearly higher although certainly not perfect. Importantly, all 4 falsepositive PET lesions, falsely overstaging the disease in 4 patients, were considered as equivocal by the blinded PET reviewer. The decision to classify these equivocal studies as positive was based on the fact that in other types of tumors these lesions sometimes do indicate the presence of a limited load of neoplastic cells. However, in our experience the clinical impact of these equivocal PET lesions is often relatively limited. Multidisciplinary confrontation of the PET findings with clinical data, together with close correlation of PET and CT images, which was not done in this study, often neutralizes the potential negative impact of these equivocal lesions. Indeed, in our study, the 4 lesions reported as equivocal on PET did not induce any change of patient management because these lesions lacked any clinical or radiologic correlation. The impact of false-positivity of PET would be more pronounced if the technique were used as an early diagnostic maneuver. In that case correlative CT imaging would frequently be necessary to decide on equivocal PET lesions.

We conclude that (1) FDG-PET allows a highly sensitive diagnosis and accurate whole-body staging of symptomatic recurrent esophageal cancer; (2) FDGPET is not accurate for diagnosis of perianastomotic recurrence owing to frequent false-positive findings based on FDG accumulation in areas of inflammation; (3) a multidisciplinary correlative approach is essential to reduce the potential negative impact of false-positive PET diagnosis on patient care; (4) prospective studies using PET in asymptomatic patients are needed to study the potential benefit of earlier diagnosis of recurrent disease on patient survival and quality of life.

\section{REFERENCES}

1. Kato H, Tachimori Y, Mizobuchi S, Igaki H, Ochiai A. Cervical, mediastinal, and abdominal lymph node dissection (three-field dissection) for superficial carcinoma of the thoracic esophagus. Cancer 1993;72:2879-82.

2. Baba M, Aikou T, Yoshinaka H, Natsugoe S, Fukumoto T, Shimazu H, et al. Long-term results of subtotal esophagectomy with three-field lymphadenectomy for carcinoma of the thoracic esophagus. Ann Surg 1994;219:310-6.

3. Nigro JJ, DeMeester SR, Hagen JA, DeMeester TR, Peters JH, Kiyabu M, et al. Node status in transmural esophageal adenocarcinoma and outcome after en bloc esophagectomy. J Thorac Cardiovasc Surg 1999;117:960-8.

4. Altorki NK, Girardi L, Skinner DB. En bloc esophagectomy improves survival for stage III esophageal cancer. J Thorac Cardiovasc Surg 1997;114:948-55.

5. van Lanschot JJ, Tilanus HW, Voormolen,MH, van Deelen RA. Recurrence pattern of oesophageal carcinoma after limited resection does not support wide local excision with extensive lymph node dissection. Br J Surg 1994;81:1320-23.

6. Fahn HJ, Wang LS, Huang BS, Huang MH, Chien KY. Tumor recurrence in long-term survivors after treatment of carcinoma of the esophagus. Ann Thorac Surg 1994;57:669-76.

7. Mantravardi RV, Lad T, Briele H, Liebner EJ. Carcinoma of the esophagus: sites of failure. Int J Radiat Oncol Biol Phys 1982;8:1897-901.

8. Law SYK, Fok M, Wong J. Pattern of recurrence after oesophageal resection for cancer: clinical implications. Br J Surg 1996;83:107-11.

9. Raoul JL, Le Prise E, Meunier B, Julienne V, Etienne PL, Gosselin M, et al. Combined radiochemotherapy for postoperative recurrence of oesophageal cancer. Gut 1995;37:174-6.

10. Carlisle JG, Quint LE, Francis IR, Orringer MB, Smick JF, Gross BH. Recurrent esophageal carcinoma: CT evaluation after esophagectomy. Radiology 1993;189:271-5.

11. Barbier PA, Luder PJ, Schupfer G, Becker CD, Wagner HE. Quality of life and patterns of recurrence following transhiatal esophagectomy for cancer: results of a prospective follow-up in 50 patients. World J Surg 1988;12:270-6.

12. Luketich JD, Friedman DM, Weigel TL, Meehan MA, Keenan RJ, Townsend DW, et al. Evaluation of distant metastases in esophageal cancer: 100 consecutive positron emission tomography scans. Ann Thorac Surg 1999;68:1133-7.

13. Block MI, Patterson GA, Sundaresan RS, Bailey MS, Flanagan FL, Dehdashti F, et al. Improvement in staging of esophageal cancer with the addition of positron emission tomography. Ann Thorac Surg 1997;64:770-7.

14. Flanagan FL, Dehdashti F, Siegel BA, Trask DD, Sundaresan SR, Patterson GA, et al. Staging of esophageal cancer with 18F-fluorodeoxyglucose positron emission tomography. AJR Am J Roentgenol 1997;168:417-24.

15. Fukunaga $\mathrm{T}$, Okazumi $\mathrm{S}$, Koide $\mathrm{Y}$, Isono $\mathrm{K}$, Imazeki $\mathrm{K}$. Evaluation of esophageal cancers using fluorine-18-fluorodeoxyglucose PET. J Nucl Med 1998;39:1002-7.

16. Flamen P, Lerut T, Stroobants S, Van Cutsem E, Dupont P, Bormans G, et al. FDG-PET for preoperative lymph node staging of esophageal cancer. J Clin Oncol 2000;18:3202-10.

17. Warburg O. On the origin of cancer cells. Science 1956;123:30914.

18. Pauwels E, McCready VR, Stoot JH, van Deurzen DF. The mechanism of accumulation of tumour-localising radiopharmaceuticals. Eur J Nucl Med 1998;25:277-305.

19. Flamen P, Stroobants S, Van Cutsem E, Dupont P, Bormans G, De Vadder N, et al. Additional value of whole-body positron emis- 
sion tomography with fluorine-18-2-fluoro-2-deoxy-D-glucose in recurrent colorectal cancer. J Clin Oncol 1999;17:894-901.

20. Lowe V, Boyd JH, Dunphy FR, Kim H, Dunleavy T, Collins BT, et al. Surveillance for recurrent head and neck cancer using positron emission tomography. J Clin Oncol 2000;18:651-8.

21. Fockens P, Manshanden CG, van Lanschot JB, Obertop H, Tytgat GNJ. Prospective study on the value of endosonographic followup after surgery for esophageal carcinoma. Gastrointest Endosc 1997;46:487-91.
22. Pierie JP, De Graaf PW, Poen H, van der Tweel I, Obertop H. Incidence and management of benign anastomotic stricture after cervical oesophagogastrostomy. Br J Surg 1993;80:471-4.

23. Nordic Gastrointestinal Tumor Adjuvant Therapy Group. Expectancy or primary chemotherapy in patients with advanced asymptomatic colorectal cancer: a randomized trial. J Clin Oncol 1992:10:904-11.

24. Strauss LG. Fluorine-18 deoxyglucose and false-positive results: a major problem in the diagnostics of oncological patients. Eur $\mathrm{J}$ Nucl Med 1996;23:1409-15.

Access to The Journal of Thoracic and Cardiovascular Surgery Online is now reserved for print subscribers!

Full-text access to The Journal of Thoracic and Cardiovascular Surgery Online is now available for all print subscribers. To activate your individual online subscription, please visit The Journal of Thoracic and Cardiovascular Surgery Online, point your browser to http://www.mosby.com/jtcvs, follow the prompts to activate your online access, and follow the instructions. To activate your account, you will need your subscriber account number, which you can find on your mailing label (note: the number of digits in your subscriber account number varies from 6 to 10). See the example below in which the subscriber account number has been circled:

\section{Sample mailing label}

\begin{tabular}{l|l|}
\cline { 2 - 3 } This is your subscription & $\begin{array}{l}* * * * * * * * * * * * * * * * * * * * * * * * * * 3-D I G I T \\
\text { account number }\end{array}$ \\
SJ P1
\end{tabular}

Personal subscriptions to The Journal of Thoracic and Cardiovascular Surgery Online are for individual use only and may not be transferred. Use of The Journal of Thoracic and Cardiovascular Surgery Online is subject to agreement to the terms and conditions as indicated online. 